\section{The effect of consistency of reward on compound-cue discrimination learning*}

\author{
T. GARY WALLER \\ University of Waterloo, Waterloo, Ont., Canada
}

Rats first were trained on a compound-cue discrimination and then were transferred to a discrimination on which one of the sets of cues continued to be relevant and the other was made variable irrelevant. The rats were either consistently rewarded (CR) during both phases, partially rewarded (PR) during both phases, or received $P R$ during acquisition and $C R$ during transfer. $C R$ during acquisition produced superior acquisition and transfer performance. Implications for stimulus-analyzer theory were indicated.

According to attention theories of discrimination learning (Sutherland, 1964; Lovejoy, 1968), organ isms must do two things in order to learn a discrimination problem. First, they must learn to attend to appropriate cues, and, second, they must learn a correct instrumental response. If an animal receives partial reward (PR) rather than consistent reward (CR) for correct responses, the theory holds that the animal attends to many cues and attaches the instrumental response to the output of many analyzers (cf. Sutherland, 1966). Thus, in a compound-cue discrimination task, PR animals are expected to demonstrate greater breadth of learning than $\mathrm{CR}$ animals.

It follows that if one of the cues in a two-cue compound is dominant and the other is nondominant (i.e., one cue is clearly more likely to be attended to than the other), PR animals should learn more about the nondominant cue than $\mathrm{CR}$ animals. Thus, $\mathrm{PR}$ animals should perform better on single-dimension tests on the nondominant cue and should perform better on a transfer task in which only the nondominant cue is relevant.

The above prediction was tested by Waller (in press) with results exactly opposite to the prediction. That is, CR animals performed better on a transfer discrimination. One purpose of the research reported here was to repeat the basic design of the earlier studies with several procedural changes. Rats were first trained on a simultaneous discrimination with both texture and brightness cues relevant. Previous results (Waller, in press) indicated that brightness was the dominant cue and texture the nondominant cue. After reaching acquisition criterion, the rats were trained on either a texture

* This research was supported by Grant A0326 from the National Research Council of Canada and by a university research grant from the University of Waterloo. Requests for reprints should be sent to $T$. Gary Waller, Department of Psychology, University of Waterloo, Waterloo, Ont., Canada. discrimination or a brightness discrimination. One group was trained with CR in both phases, while a second group received $P R$ in acquisition and $C R$ in transfer. In order to assess the effect of the shift from PR to $C R$, a third group was trained with $P R$ in both phases. Since previous research demonstrated that texture was the nondominant cue, stimulus-analyzer theory predicts that PR animals should learn more about the texture dimension than $C R$ animals. The present research differed from the earlier studies (1) in the inclusion of the group trained on PR throughout, (2) in the use of a noncorrection procedure rather than a modified correction procedure, and (3) in the fact that there was no interpolated CR phase at the end of discrimination training for the PR animals.

\section{SUBJECTS}

The Ss were 48 experimentally naive male albino rats supplied by the Holtzman Co. On arrival at the laboratory, average weight was approximately $290 \mathrm{~g}$ and average age was reported to be 90-100 days.

$$
\text { APPARATUS }
$$

The apparatus was described previously (Waller, in press) and was an enclosed wooden single-unit T-maze which was painted flat gray and covered with Plexiglas. Reward pellets were placed in gray foodcups behind a wooden barrier at the rear of the goalboxes. A guillotine door separated the startbox from the stem; horizontally sliding wooden doors separated the choice area from each goalbox. Stimulus inserts for the floors of the arms of the maze were cut from Masonite and were painted flat white (W) or black (B) to define a brightness dimension. For the texture dimension, a smooth floor (S) was defined as the smooth side of painted Masonite; a rough floor $(R)$ was devised by drilling $0.5-\mathrm{cm}$ holes centered every $1.25 \mathrm{~cm}$ over the length and breadth of the Masonite insert. Each insert extended from the center of the choice area to the foodcup.

\section{PROCEDURE}

On arrival, the rats were housed individually and placed on ad lib food and water until the fourth day, at which time all food was removed from the cages. Beginning on Day 5, the daily ration was controlled to maintain a constant $85 \%( \pm 10 \mathrm{~g})$ of terminal ad lib weight throughout the experiment. Beginning on Day 5 and continuing for 8 days, 2-min prehandling sessions were given daily to each rat. During each prehandling session the rat was picked up and replaced at least three times by $E$, was permitted to explore the top of a large gray table, and was given access to five $.045 \cdot \mathrm{g}$ food pellets located in a glass dish in the center of the table.

\section{Acquisition}

Beginning on Day 13 , each rat was given acquisition training on a simultaneous discrimination in which brightness and texture were relevant and redundant cues. No attempt was made to counterbalance completely for positive cue (cf. Sutherland \& Holgate, 1966), in that all rats were rewarded for choosing the white floor. For a randomly selected half of the rats, the white-smooth (WS) floor was positive (S+) and black-rough (BR) was negative (S-); for the other half, WR was $\mathrm{S}+$ and BS was S-. Training was with a noncorrection procedure, and the right-left position of $\mathrm{S}+$ was determined by randomly selected Gellermann series. Each rat received 16 trials/day to a criterion of 15 correct responses on any 16 consecutive trials. When appropriate, correct responses were rewarded with five $.045-\mathrm{g}$ Noyes pellets. Errors were never rewarded, but the foodcup was always present in the incorrect goalbox. The within-day intertrial interval was approximately $10 \mathrm{sec}$. Rats in Group CC were rewarded for every correct response; rats in Groups PC and PP were rewarded for a randomly selected $50 \%$ of the correct responses in every block of 10 , except that the first two correct responses were rewarded for every rat.

$$
\text { Transfer }
$$

On the day after each $S$ reached acquisition criterion, it was shifted to a discrimination in which one of the acquisition dimensions continued to be relevant and $\mathrm{S}+$ on that dimension was unchanged. The other previously relevant dimension was changed to variable-between irrelevant such that only one value of the irrelevant dimension was present on any single trial. For example, if $S+$ was WS and $S-$ was BR during acquisition, $S$ was shifted to either (1) a brightness discrimination in which WS-BS appeared on half the trials, WR-BR appeared on the other half, and WS and WR were $S+$, or (2) a texture discrimination in which WS-WR or 
BS-BR appeared equally often and S+ was WS and BS. Rats in Groups CC and PC were rewarded for every correct response during transfer; those in Group PP were rewarded for a randomly selected $50 \%$ of the correct responses. All other procedures were the same as in acquisition.

RESULTS AND DISCUSSION

Mean trials to acquisition criterion for Groups CC, PC, and PP were $68.25,117.31$, and 115.81 , respectively; mean errors for white-smooth positive and white-rough positive were 81.75 and 119.17, respectively. Analysis of variance indicated that the effect of reward condition was significant ( $F=5.36$, $\mathrm{df}=2 / 42, \mathrm{p}<.01$ ) and that learning was faster with smooth positive $(F=7.22$, df $=1 / 42, p<.025)$, but that the interaction of reward condition with positive cue was not significant $(\mathrm{F}<1.0)$. Individual comparisons by the Newman-Keuls procedure (Winer, 1962) indicated that Group CC learned faster than Group PC $(q=5.76, \quad \mathrm{df}=3 / 42$, $\mathrm{p}<.01)$ or Group PP $(\mathrm{q}=5.58$, $\mathrm{df}=2 / 42, p<.01$ ), but that Groups $P C$ and PP did not differ significantly during acquisition $(q<1.0)$. The acquisition data confirm the essential aspect of earlier results (Waller, in press; Cranford \& Clayton, 1970; Erlebacher, 1963) in demonstrating that CR produces superior performance to $\mathrm{PR}$ during acquisition of a simultaneous discrimination task.

Mean trials to transfer criterion as a function of reward condition and relevant dimension during transfer are shown in Table 1. Group CC learned fastest, followed by Groups PC and PP, and performance was superior on

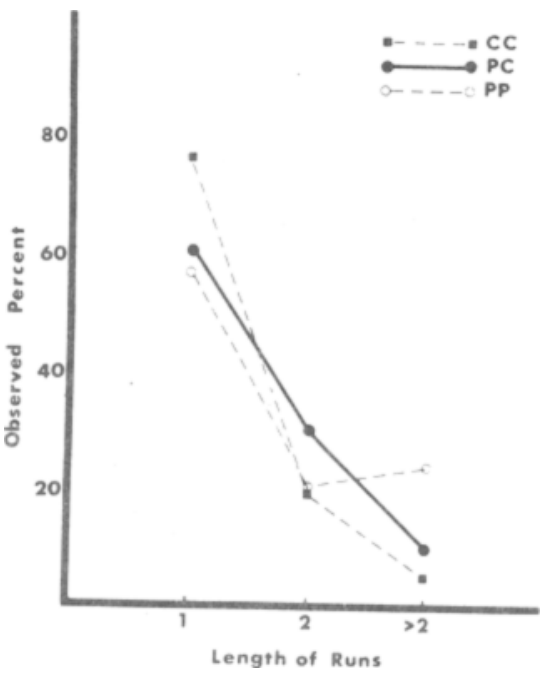

Fig. 1. Observed percentage of error runs of Lengths 1,2 , or greater than 2 for Groups CC, PC, and PP.

Table 1

Mean Trials to Criterion During Transfer

Relevant Dimension During Transfer

Percentage of Reward During

Acquisition and Transfer

Consistent-Consistent

Partial-Consistent

Partial-Partial
Brightness

15.38

19.00

22.88
Texture

24.12

44.37

57.75 the brightness discrimination for all reward conditions. Analysis of variance of trials to criterion indicated that the effect of reward condition was significant $(\mathrm{F}=3.33, \mathrm{df}=2 / 36$, $\mathrm{p}<.05$ ), as was the effect of relevant dimension $(\mathrm{F}=12.36, \mathrm{df}=1 / 36$, $\mathrm{p}<.01$ ), but that neither the interaction of reward condition with relevant dimension ( $F=1.35$, $\mathrm{df}=2 / 36$ ) nor any effects involving positive cue (all $F<1.0$ ) were significant. Individual comparisons with the Newman-Keuls procedure showed that Group CC learned faster than Group PC $(\mathrm{q}=4.12, \mathrm{df}=2 / 36$, $\mathrm{p}<.01)$ or Group PP $(q=7.22$, $\mathrm{df}=3 / 36, p<.01)$ and that Group PC learned faster than Group PP $(q=3.09, \quad$ df $=2 / 36, \quad p<.05)$. Although the interaction of reward condition with relevant dimension was not significant, previous research indicated that reward conditions would differentially affect the two transfer tasks, so the simple effects of reward condition for each relevant dimension were evaluated. Reward conditions did not significantly affect performance on the brightness task $(F<1.0)$ but did have a significant effect on the texture task $(F=4.42$, $\mathrm{df}=2 / 36, \mathrm{p}<.05$ ). Individual comparisons on the texture task indicated that Group CC performed better than Group PC $(q=3.56$, $\mathrm{df}=2 / 36, \quad \mathrm{p}<.05)$ or Group PP $(q=5.90, d f=3 / 36, p<.01)$ but that Groups $P C$ and $P P$ were not reliably different from each other $(q=2.35$, $\mathrm{df}=2 / 36$ ).

The transfer results substantiated earlier research (Waller, in press) in demonstrating that (1) the brightness discrimination was easier than the texture discrimination, (2) transfer performance on the easier brightness task was affected only slightly, if at all, by percentage of reward during acquisition, and (3) transfer performance on the more difficult texture discrimination was significantly retarded by $P R$ during acquisition of the compound-cue discrimination. The impetus for the research previously reported and for the research reported here was a prediction from stimulus-analyzer theory of discrimination learning (Sutherland, 1964, 1966; Lovejoy, 1968), i.e., that in a discrimination situation where one of two compound cues is dominant (brightness) and one is nondominant (texture), $\mathrm{PR}$ animals should learn more about the nondominant cue than would $\mathrm{CR}$ a nimals, and, on transfer to a discrimination which retained the nondominant texture cues, the PR animals should perform better than the CR animals. That is, Group PC should perform better in transfer than Group CC. A significant effect in the opposite direction was observed, thus confirming the previous lack of support for the above prediction. One possible explanation of the earlier result, where a period of $C R$ was given to $P R$ animals at the end of acquisition on the compound cues, was that the interpolated $\mathrm{CR}$ phase served as overtraining. Sutherland \& Holgate (1966) showed that overtraining with CR on a compound-cue task with a dominant and nondominant cue increased the amount learned about the less salient cue. Thus, in the earlier research the superiority of $C R$ animals on the texture discrimination possibly could be attributed to overtraining on the compound. However, there was no interpolated $C R$ in the research reported here and performance on texture transfer was superior for CR animals.

The present research demonstrated that the shift from PR to $C R$ (Group PC) did not produce discrimination performance equal to performance for rats trained on $\mathrm{CR}$ in both phases (Group CC). While Group PC showed some improvement as compared to Group PP, the improvement was not statistically reliable. Thus the results are not in agreement with performance in the runway, where it has been shown (Theios \& McGinnis, 1967) that rats shifted from PR to CR perform under $C R$ as well as rats trained throughout under $C R$. The failure on the part of Group PC to perform as well as Group CC on the second discrimination task indicates that performance on discrimination tasks is subject to the influence of previous reward contingencies. The effect of $P R$ during acquisition on transfer performance is at least partly attibutable to the effect of inconsistent reward on lengths of error runs. The percentage of error runs that were of lengths 1,2 , or greater than 2 are shown in Fig. 1 for all three groups.

The lengths of error runs were 
dependent upon reward conditions $\left(x^{2}=21.64, \mathrm{df}=4, \mathrm{p}<.01\right)$ in that animals receiving $C R$ during acquisition made fewer error runs of lengths greater than 1 , and animals receiving $P R$ during acquisition made more error runs of lengths greater than 1. While the tendency to make longer error runs was less for Group PC than for Group PP, both groups PC and PP made more long runs of errors than did Group CC. The tendency for PR animals to make more of the longer error runs suggests that nonreward provided less information or had less value as a cue for PR animals (Groups
CP and PP) than for CR animals.

\section{REFERENCES}

CRANFORD, J. L.. \& CLAYTON, K. N. Effects of percentage of reward and amount of stimulus exposure on compound-cue discrimination learning by rats. Journal of Comparative \& Physiological Psychology, 1970, 71. 497-502.

ERLEBACHER, A. Reversal learning in rats as a function of percentage of reinforcement and degree of learning. Journal of Experimental Psychology, $1963,66,84-90$.

LOVEJOY E. P. Attention in discrimination learning. San Francisco: Holden-Day, 1968.

SUTHERLAND. $N$. S. The learning of discriminations by animals. Endeavour,
$1964,23,148-152$.

SUTHERLAND, N. S. Partial reinforcement and breadth of leaming. Quarterly Journal of Experimental Psychology. 1966, 18, 289-301.

SUTHERLAND, N. S., \& HOLGATE, V. Two-cue discrimination learning in rats. Journal of Comparative \& Physiological Psychology, 1966, 61, 198-207.

THEIOS, J., \& McGINNIS, R. W. Partial reinforcement before and after continuous reinforcement. Journal of Experimental Psychology, 1967, 73, 479-481.

WALLER, T. G. The effect of percentage of reward on compound-cue discrimination learning by rats. Learning \& Motivation, 1971 , in press.

WINER, B. J. Statistical principles in experimental design. New York: McGraw-Hill, 1962.

\section{CURRENT LITERATURE ON REINFORCEMENT IN ANIMALS}

DARDANO, J. F. (Division of Behavioral Biology, Johns Hopkins University School of Medicine, Baltimore, Md. 21205). Control of concurrent avoidance and appetitive behaviors by an indicator of shock proximity. Journal of the Experimental Analysis of Behavior, $1971,15,167-180$.

DAVIDSON, A. B. (Research and Development Division, Smith Kline \& French Laboratories, Philadelphia, Pa. 19101), DAVIS, D. J., \& COOK, L. A rapid automatic technique for generating operant key-press behavior in rats. Journal of the Experimental Analysis of Behavior, 1971, 15, 123-127.

ELLEN, P. (Georgia State University, Atlanta, Ga. 30303), \& AITKEN, W. C., JR. Absence of temporal discrimination following septal lesions. Psychonomic Science, 1971, 22, 129-131.

FRANCHINA, J. J. (College of Arts and Sciences, Virginia Polytechnic Institute, Blacksburg, Va. 24061), SPARLING, D. L., \& CHLIPALA, R. Effects of patterns of goal-box placements on the subsequent acquisition of instrumental response patterning. Journal of Comparative and Physiological Psychology, 1971, 74, 84-89.

HOMZIE, M. J. (University of Virginia, Charlottesville, Va. 22901), GOHMANN, T., \& HALL, S. W., JR. Runway performance in rats as determined by the predictive value of intertrial reinforcements. Journal of Comparative and Physiological Psychology, 1971, 74,
90-95.

KRASNEGOR, N. A. (Department of Experimental Psychology, Division of Neuropsychiatry, Walter Reed Army Institute of Research, Walter Reed Army Medical Center, Washington, D.C. 20012), BRADY, J. V., \& FINDLEY, J. D. Second-order optional avoidance as a function of fixed-ratio requirements. Journal of the Experimental Analysis of Behavior, $1971,15,181-187$.

ROUTTENBERG, A. (Northwestern University, Evanston, Ill. 60201). Forebrain pathways of reward in Rattus norvegicus. Journal of Comparative and Physiological Psychology, 1971, 75, 269-276.

SCHOEL, W. M. (University of South Florida, Tampa, Fla. 33620), DAVIS, J., \& BITTERMAN, M. E. Adventitious reinforcement in free-operant discrimination. Behavior Research Methods \& Instrumentation, 1971, 3, 8-9.

SHAPIRO, M. M. (Emory University, Atlanta, Ga. 30322), MUGG, G. J., \& EWALD, W. Instrumental preferences and conditioned preparatory responses in dogs. Journal of Comparative and Physiological Psychology, 1971, 74, 227-232.

SHAPIRO, M. M. (Emory University, Atlanta, Ga. 30322), SADLER, E. W., \& MUGG, G. J. Compound stimulus effects during higher order salivary conditioning in dogs. Journal of Comparative and Physiological Psychology, 1971, 74, 222-226.

SHIMP, C. P. (University of Utah, Salt
Lake City, Utah 84112), \& WHEATLEY, $K$. L. Matching to relative reinforcement frequency in multiple schedules with a short component duration. Journal of the Experimental Analysis of Behavior, $1971,15,205-210$.

SHULL, R. L. (University of North Carolina at Greensboro, Greensboro, N.C. 27412). Sequential patterns in post-reinforcment pauses on fixed-interval schedules of food. Journal of the Experimental Analysis of Behavior, 1971, 15, 221-231.

TANG, M., \& COLLIER, G. (Rutgers-The State University, New Brunswick, N.J. 08903). Effect of successive deprivations and recoveries on the level of instrumental performance in the rat. Journal of Comparative and Physiological Psychology, 1971, 74, 108-111.

WEINSTOCK, R. B. (Mary Washington College of the University of Virginia, Fredericksburg, Va. 22401). Preacquisition exploration of the runway in the determination of contrast effects in the rat. Journal of Comparative and Physiological Psychology, 1971, 75, 107-115.

WILLIAMS, B. A. (Colorado College, Colorodo Springs, Colo. 80903). Color alternation learning in the pigeon under fixed-ratio schedules of reinforcement. Journal of the Experimental Analysis of Behavior, $197115,129-140$. 